\title{
AN EMBEDDING OF PI-RINGS
}

\section{A. S. AMITSUR}

1. Introduction. It is well known that a commutative ring which has no nonzero nilpotent ideals is isomorphic to a subring of a complete direct sum of commutative fields ( $\mathrm{McCoy}[1]) .{ }^{1}$ In this note, this fact is generalised to rings which satisfy a polynomial identity (PI-rings). We show that every PI-ring which has no nilpotent ideals ${ }^{2}$ is isomorphic to a subring of a complete direct sum ${ }^{8}$ of central simple algebras whose order over their centre is bounded. As a consequence we prove that these rings are subrings of matrix rings over commutative rings. This implies an extension of a result of [2] concerning the minimal identity of a simple algebra. We prove that for a PI-ring which has no nonzero nilpotent ideals, the standard identity $S_{d}(x)$ $=0$, where $d$ is an even integer, is the unique (up to a numerical factor) minimal identity which is linear in each of its indeterminates. The term standard identity was ascribed in [2] to the polynomial identity:

$$
S_{d}(x)=S_{d}\left(x_{1}, \cdots, x_{d}\right)=\sum_{(i)} \pm x_{i_{1}} \cdots x_{i_{d}}=0
$$

where the sum ranges over all permutations ( $i$ ) of $d$ letters, and the sign is positive for even permutations and negative for odd permutations.

Notations. A polynomial identity of minimum degree satisfied by a PI-ring $R$ will be called a minimal identity of $R$. We shall refer to a polynomial identity which is linear and homogeneous in each of its indeterminates as a linear identity. We shall use the following three types of semi-simplicity: a ring $R$ is said to be

(a) J-semi-simple, if $R$ is semi-simple in the sense of Jacobson [3], that is, if the quasi-regular radical of $R$ is zero.

(b) $\mathrm{K}$-semi-simple, if $R$ does not contain any nonzero nil ideals.

(c) A-semi-simple, if $R$ has no nonzero nilpotent ideals.

2. The ring $R[x]$. We denote by $R[x]$ the ring of all polynomials in the commutative indeterminate $x$ over $R$. In this section we deal with properties of $R[x]$ induced by $R$.

Received by the editors January 4, 1951.

${ }^{1}$ Numbers in brackets refer to the bibliography at the end of the paper.

2 Ideals will always mean two-sided ideal.

${ }^{3}$ For definition of (complete) direct sums and of subdirect sums see, for example, $[1$, p. 121]. 
Lemma 1. Let $P$ be a nonzero ideal in $R[x]$ and let $p(x)=a_{0}+\ldots$ $+a_{n} x^{n}\left(a_{n} \neq 0\right)$ be a polynomial of minimum degree in $P$. Then if $b \in R$ such that $a_{n}^{\mu} b=0$ for some integer $\mu$, then $a_{n}^{\mu-1} p(x) b=0$.

Indeed, the coefficient of $x^{n}$ in $a_{n}^{\mu-1} p(x) b \in P$ is $a_{n}^{\mu} b=0$, that is, this polynomial is of lower degree than that of $p(x)$. Hence the minimality of the degree of $p(x)$ implies that $a_{n}^{\mu-1} p(x) b=0$.

CoRollary. If $r(x) \in R[x]$ such that $a_{n}^{\mu} r(x)=0$ for some integer $\mu$, then $a_{n}^{\lambda} p(x) r(x)=0$ for every integer $\lambda \geqq \mu-1$.

This follows immediately by the preceding lemma, since each of the coefficients of $r(x)$ satisfies the condition of that lemma.

We prove now the following fundamental lemma:

Lemma $2{ }^{4}$ If $R$ is a $\mathrm{K}$-semi-simple ring, then $R[x]$ is $\mathrm{J}$-semi-simple.

Proof. Assume that $R[x]$ is not J-semi-simple. Denote by $J_{x}$ the nonzero Jacobson's radical of $R[x]$. It is readily verified that the totality of the coefficients of the highest power of the polynomials of $J_{x}$ of degree $n$-where $n$ is the minimal degree of the nonzero polynomials of $J_{x}-$ constitute a nonzero ideal in $R$. The lemma will be proved if it is shown that this ideal is a nil ideal, that is, that if $p(x)=a_{0}+a_{1} x+\cdots+a_{n} x^{n}$ is a nonzero polynomial of minimum degree in $J_{x}$, then $a_{n}^{\mu}=0$ for some integer $\mu$.

To this end we consider the polynomial $p(x) x a_{n}$ (which belongs to $J_{x}$, since $p(x) \in J_{x}$ and $\left.x a_{n} \in R[x]\right)$ and its quasi-inverse $q(x)$. By Lemma 1 of [3] and Theorem 2 of [3] it follows that

$$
\begin{aligned}
& p(x) x a_{n}+q(x)+p(x) x a_{n} q(x)=0, \\
& p(x) x a_{n}+q(x)+q(x) p(x) x a_{n}=0 .
\end{aligned}
$$

By (1) we obtain that $q(x)=x t(x),{ }^{5} t(x) \in R[x]$. Put $s(x)=p(x) a_{n}$. Then (1) implies that $x s(x)+x t(x)+x^{2} s(x) t(x)=0$. Hence, ${ }^{6}$

$$
s(x)+t(x)+x s(x) t(x)=0 .
$$

Similarly, we obtain from (2) that

$$
s(x)+t(x)+x t(x) s(x)=0 .
$$

Suppose $a_{n}^{\mu} t(x) \neq 0$ for every integer $\mu$. Let $\nu$ be the minimal degree of the polynomials $a_{n}^{\mu} t(x)$. Write

\footnotetext{
4 If $R$ is commutative, this lemma is a consequence of [7, Corollary 8.1].

- If $R$ does not possess a unit and $x \notin R[x]$, we adopt the notation $x t(x)$ (similarly $t(x) x)$ for the polynomial $x b_{0}+\cdots+x^{n+1} b_{n}$, where $t(x)=b_{0}+\cdots+x^{n} b_{n}$.

- Since $x m(x)=0$ if and only if $m(x)=0$.
} 


$$
t(x)=t_{1}(x)+x^{\nu+1} t_{2}(x),
$$

where $t_{1}(x)=b_{0}+b_{1} x+\cdots+b_{\nu} x^{\nu}$. The minimality of $\nu$ implies that

$$
a_{n}^{\mu} b_{\nu} \neq 0 \quad \text { for every integer } \mu,
$$

and

(7) $\quad a_{n}^{\mu} t_{2}(x)=0$ for every $\mu$ greater than some integer $\pi$.

The polynomial $s(x)=p(x) a_{n}$ is of minimum degree in $J_{x}$, and its highest coefficient is $a_{n}^{2}$. Hence, since $a_{n}^{2 \mu} t_{2}(x)=0$ (for $\mu \geqq \pi$ ), it follows by the corollary of Lemma 1 that

$$
a_{n}^{\mu} s(x) t_{2}(x)=0 \quad \text { for every } \mu \geqq 2 \pi .
$$

Substituting (5) into (3) and multiplying this equation on the left by $a_{n}^{\lambda}$, where $\lambda=2 \pi$, we obtain, by (7) and (8),

$$
a_{n}^{\lambda} s(x)+a_{n}^{\lambda} t_{1}(x)+x a_{n}^{\lambda} s(x) t_{1}(x)=0 .
$$

The degree of both $a_{n}^{\lambda} s(x)$ and $a_{n}^{\lambda} t_{1}(x)$ is less than $n+\nu+1$, and the coefficient of $x^{n+\nu+1}$ of $x a_{n}^{\lambda} s(x) t_{1}(x)$ is $a_{n}^{\lambda+2} b_{\nu}$. Hence $a_{n}^{\lambda+2} b_{\nu}=0$. But this contradicts (6); hence our assumption that $a_{n}^{\mu} t(x) \neq 0$, for every integer $\mu$, is false. Thus $a_{n}^{\lambda} t(x)=0$ for some integer $\lambda$. Now multiplication of (4) on the left by $a_{n}^{\lambda}$ yields $a_{n}^{\lambda} s(x)=0$; hence $a_{n}^{\lambda+2}=0$, q.e.d.

\section{A-semi-simple PI-rings.}

Lemma 3. If $R$ is a PI-ring, then $R[x]$ is also a PI-ring, and the totalities of the linear-identities of $R$ and $R[x]$, respectively, coincide.

The first part of the lemma follows from the fact that $R$ satisfies a linear identity (Lemma 2 of [4]), and this identity is evidently satisfied by $R[x]$. If we assume that the operators of $R$, which are the coefficients of the identities of $R$, were extended to operate on $R[x]$ by defining $\alpha\left(\sum a_{\nu} x^{\nu}\right)=\sum\left(\alpha a_{\nu}\right) x^{\nu}$, the rest of the lemma is readily verified.

The following lemma follows immediately:

LEMMA 4. $A$ necessary and sufficient condition that a subdirect sum of a set of $P I$-rings $\left\{Q_{\alpha}\right\}$ satisfies an identity $F\left(x_{1}, \cdots, x_{m}\right)=0$ is that each of the rings $Q_{\alpha}$ satisfies the identity $F=0$.

We recall that a PI-ring $R$ is said to be of degree $d[5]$ if $d$ is the minimal degree of the polynomial identities satisfied by $R$.

REMARK. It has been shown in [2] that a central simple algebra $A$ of order $n^{2}$ over its centre is a PI-ring of degree $2 n$, and the minimal 
linear-identity of $A$ is the standard identity $S_{2 n}(x)=0$, uniquely determined up to a numerical factor. Evidently, $A$ satisfies also the identities $S_{n}(x)=0$ for every $m \geqq 2 n .^{7}$

We prove now:

TheOREM 1. If $R$ is a J-semi-simple PI-ring of degree $d$, then

(1) $d=2 m$.

(2) The ring $R$ is a subdirect sum of a set of central simple algebras $\left\{A_{\alpha}\right\}$ such that $m^{2}$ is the upper bound of the orders of these algebras over their centres.

(3) The standard identity $S_{d}(x)=0$ is the unique (up to a numerical factor) minimal linear-identity of $R$.

Proof. Since $R$ is J-semi-simple, $R$ is a subdirect sum of primitive rings $\left\{A_{\alpha}\right\}$ (Theorem 28 of [3]), Lemma 4 implies that each $A_{\alpha}$ is a PI-ring of degree not greater than $d$. Hence, by Theorem 1 of [4] and by consequence 2 of [5] it follows that each $A_{\alpha}$ is a central simple algebra of order not greater than $[d / 2]^{2}$. Let $m^{2}$ be the upper bound of the orders of the algebras $A_{\alpha}$; then $m \leqq[d / 2]$. By the preceding remark it follows that each $A_{\alpha}$ satisfies the identity $S_{2 m}(x)=0$. Thus, Lemma 4 implies that this identity is satisfied, as well, by their subdirect sum $R$; hence, $d \leqq 2 m$. On the other hand, $2 m \leqq 2[d / 2]$ $\leqq d$. Hence $m=[d / 2]$ and $d=2 m$. This completes the proof of the first two parts of the theorem. Since the upper bound $m^{2}$ is achieved by some $A_{\beta}$, and the minimal identities of $R$, whose degree is $2 m$, are also identities of this algebra, the proof of the third part of our theorem follows immediately by the preceding remark, that is, by Theorem 7 of [2].

We turn now to the main theorem of this paper:

Theorem 2. Let $R$ be an A-semi-simple PI-ring of degree $d$, then

(1) $d=2 m$.

(2) The ring $R$ is a subring of a complete direct sum of central simple algebras $\left\{A_{\alpha}\right\}$ such that $m^{2}$ is the upper bound of the orders of these algebras over their centres.

(3) The identity $S_{d}(x)=0$ is the unique (up to a numerical factor) minimal linear-identity of $R$.

Proof. Since $R$ is a PI-ring which is A-semi-simple, the corollary of Theorem 4 of [5] implies that $R$ is also $\mathrm{K}$-semi-simple; hence by Lemma 2 it follows that $R[x]$ is $\mathrm{J}$-semi-simple.

In the light of Lemma 3, the application of the preceding theorem to the ring $R[x]$ yields the first and the third parts of the theorem.

7 Compare with Remark 6 of [2]. 
The rest of the theorem follows now immediately from the preceding theorem since $R$ is a subring of $R[x]$ which is, by Lemma 3, a PIring of degree $d$.

Let $R[x]$ be a subdirect sum of the central simple algebras $\left\{A_{\alpha}\right\}$. By Lemma 4 it follows that the set of the identities satisfied by every $A$ coincides with the set of the identities of the complete direct sum $\sum A_{\alpha}$ as well as with the totality of the identities of $R[x]$. Hence we obtain, by Lemma 3, the following corollary.

COROLlaRY 1. The set of the linear identities of the PI-ring $R$ is the same as the set of the linear identities of the complete direct sum $\sum A_{\alpha}$.

Let $\left\{A_{\alpha}\right\}$ be a set of central simple algebras of orders not greater than $m^{2}$. Then each of these algebras satisfies the identity $S_{2 m}(x)=0$. Lemma 3 implies, therefore, that the complete direct sum $\sum A_{\alpha}$ satisfies the same identity $S_{2 m}(x)=0$. A combination of this fact and the preceding theorem yields:

Corollary 2. A necessary and sufficient condition for an A-semisimple ring to satisfy a polynomial identity is that it be isomorphic to a subring of a complete direct sum of central simple algebras of bounded order.

Another immediate consequence of the preceding theorem is:

COROLlaRY 3. Every PI-ring of odd degree contains nonzero nilpotent ideals.

Consider the ring $R$ and the central simple algebras $A_{\alpha}$ of Theorem 2. Let $F_{\alpha}$ be a splitting field of the algebra $A_{\alpha}$. Then $A_{\alpha}$ is isomorphic with a subring of the total matrix algebra $F_{\alpha m}$ of order $m^{2}$ over $F_{\alpha}$. The complete direct sum $\sum F_{\alpha m}$ of the matrix algebra $\left\{F_{\alpha m}\right\}$ contains, therefore, a subring isomorphic with the complete direct sum $\sum A_{\alpha}$. Thus it follows by Theorem 2 that $R$ is isomorphic with a subring of $\sum F_{\alpha m}$. It is readily verified that $\sum F_{\alpha m}$ is isomorphic with the total matrix ring $F_{m}$ of order $m^{2}$ over the complete direct sum $F=\sum F_{\alpha}$ of the fields $\left\{F_{\alpha}\right\}$. Since $F$ is a direct sum of fields, $F$ is a commutative A-semi-simple ring. Hence, we obtain:

Theorem $3 .^{8}$ If $R$ is a PI-ring of degree $d$ without nilpotent ideals, then $d=2 m$ and $R$ is isomorphic with a subring of a total matrix ring of order $m^{2}$ over a commutative ring which does not contain nilpotent ideals.

8 This result has been pointed out to me by the referee. 
Let $R$ be a subring of a total matrix ring of order $m^{2}$ over a commutative ring. By the proof of [2, Theorem 1] it follows that $R$ is a PI-ring which satisfies the identity $S_{2 m}(x)=0$. Hence, a combination of this fact and the preceding theorem yields:

CoRollary. An A-semi-simple ring $R$ is a PI-ring if and only if $R$ is isomorphic with a subring of a total matrix ring over a commutative ring.

4. Identities for PI-rings. Denote by $N=N(R)$ the radical of the PI-ring $R$, that is, the join of all nilpotent ideals of $R$.

In this section we apply the preceding results to obtain identities satisfied by the quotient ring $R / N(R) .^{9}$

Let $R$ be a PI-ring of degree $d$, and let $U(R)$ denote the lower radical of $R$. Since $R / U(R)$ is an A-semi-simple PI-ring, it follows by Theorem 2 , that:

TheOREM 4. If $R$ is a PI-ring of degree $d$, and $U(R)$ is the lower radical of $R$, then $R / U(R)$ satisfies the identity $S_{2 m}(x)=0$, where $2 m \leqq d$.

TheOREM 5. Let $R$ be a PI-ring of degree $d$ such that its radical $N(R)$ is a nilpotent ideal of index not greater than $\rho$, then $S$ satisfies the identity

$$
\prod_{i=1}^{\rho} S\left(x_{i_{1}}, \cdots x_{i_{d}}\right)=0
$$

Proof. The condition of the theorem implies that $U(R)=N(R)$. Hence, by the preceding theorem, $R / N(R)$ satisfies each of the identities $S\left(x_{i 1}, \cdots, x_{i d}\right)=0$. Since $N(R)^{\rho}=0$, it is readily seen that $R$ satisfies the identity (9).

By Theorem 2 of [6] it follows that the radical of the quotient ring $R / N(R)$, where $R$ is a PI-ring of degree $d$, is a nilpotent ideal of index not greater than $[d / 2]$. Hence we have the following corollary.

Corollary. If $R$ is a PI-ring of degree d, then $R / N(R)$ satisfies the identity $\prod_{i=1}^{[d / 2]} S\left(x_{i 1}, \cdots, x_{i d}\right)=0$.

In a process similar to that of the Laplace expansion of determinants one can readily prove that

$$
S_{n}\left(x_{1}, \cdots, x_{n}\right)=\sum \pm S_{k}\left(x_{i_{1}}, \cdots, x_{i_{k}}\right) S_{n-k}\left(x_{i_{k+1}}, \cdots, x_{i_{n}}\right)
$$

where the sum ranges over all $C_{n, k}$ different selections of $k$ letters $i_{1}, \cdots, i_{k}$ out of $n$ letters, and where $i_{k+1}, \cdots, i_{n}$ denotes the complement of the set $i_{1}, \cdots, i_{k}$. This readily implies that the standard

\footnotetext{
${ }^{9}$ Compare with Theorem 9 and its remark of [2].
} 
identity $S_{p q}(x)=0$ can be expressed as a sum of a set of $q$ products of standard identities each of which is of degree $p$. Hence by the preceding corollary it follows that:

Theorem 6. If $R$ is a PI-ring of degree $d$, then $R / N(R)$ satisfies the standard identity $S_{p}(x)=0$, where $p=d[d / 2]$.

\section{BIBLIOGRAPHY}

1. N. H. McCoy, Rings and ideals, Carus Monograph, no. 8, 1948.

2. A. S. Amitsur and J. Levitzki, Minimal identities for algebras, Proceedings of the American Mathematical Society vol. 1 (1950) pp. 449-463.

3. $\mathrm{N}$. Jacobson, The radical and semi-simplicity for arbitrary rings, Amer J. Math. vol. 67 (1945) pp. 300-341.

4. I. Kaplansky, Rings with a polynomial identity, Bull. Amer. Math. Soc. vol. 54 (1948) pp. 575-580.

5. J. Levitzki, $A$ theorem on polynomial identities, Proceedings of the American Mathematical Society vol. 1 (1950) pp. 334-341.

6. A. S. Amitsur, Nil PI-rings, Proceedings of the American Mathematical Society vol. 2 (1951) pp. 538-540.

7. E. Snapper, Completely primary rings I, Ann. of Math. vol. 52 (1950) pp. 666693.

HEBREW UNIVERSITY 